\title{
Cubic non-Cayley vertex-transitive bi-Cayley graphs over a regular $p$-group
}

\author{
Jin-Xin Zhou, Yan-Quan Feng \\ Department of Mathematics \\ Beijing Jiaotong University \\ Beijing 100044, P.R. China \\ jxzhou@bjtu.edu.cn, yqfeng@bjtu.edu.cn \\ Submitted: Dec 19, 2013; Accepted: Aug 6, 2016; Published: Aug 19, 2016 \\ Mathematics Subject Classifications: 05C25, 20B25
}

\begin{abstract}
A bi-Cayley graph is a graph which admits a semiregular group of automorphisms with two orbits of equal size. In this paper, we give a characterization of cubic nonCayley vertex-transitive bi-Cayley graphs over a regular $p$-group, where $p>5$ is a prime. As an application, a classification of cubic non-Cayley vertex-transitive graphs of order $2 p^{3}$ is given for each prime $p$.
\end{abstract}

Keywords: bi-Cayley graph; vertex-transitive; automorphism group; Cayley graph

\section{Introduction}

All groups considered in this paper are finite, and all graphs are finite, connected, simple and undirected. For the group-theoretic and graph-theoretic terminology not defined here we refer the reader to $[2,18]$.

A graph is said to be a bi-Cayley graph over a group $H$ if it admits $H$ as a semiregular automorphism group with two orbits of equal size. (Some authors have used the term semiCayley instead $[9,8,4,13]$. In this paper, we follow [11] to use the term bi-Cayley.) Note that every bi-Cayley graph admits the following concrete realization. Let $R, L$ and $S$ be subsets of a group $H$ such that $R=R^{-1}, L=L^{-1}$ and $R \cup L$ does not contain the identity element of $H$. Define the graph $\operatorname{BiCay}(H, R, L, S)$ to have vertex set the union of the right part $H_{0}=\left\{h_{0} \mid h \in H\right\}$ and the left part $H_{1}=\left\{h_{1} \mid h \in H\right\}$, and edge set the union of the right edges $\left\{\left\{h_{0}, g_{0}\right\} \mid g h^{-1} \in R\right\}$, the left edges $\left\{\left\{h_{1}, g_{1}\right\} \mid g h^{-1} \in L\right\}$ and the spokes $\left\{\left\{h_{0}, g_{1}\right\} \mid g h^{-1} \in S\right\}$ (Note that some authors label the vertices of a bi-Cayley graph for a group $H$ with ordered pairs $(h, i)$ for $h \in H$ and $i \in\{0,1\}$, while we are using $h_{i}$ to denote $(h, i))$. For the case when $|S|=1$, the bi-Cayley graph $\operatorname{BiCay}(H, R, L, S)$ is also called one-matching bi-Cayley graph (see [11]). Also, if $|R|=|L|=s$, then $\operatorname{BiCay}(H, R, L, S)$, is 
said to be an s-type bi-Cayley graph, and if $H$ is abelian, then $\operatorname{BiCay}(H, R, L, S)$ is simply called an abelian bi-Cayley graph.

In the study of bi-Cayley graphs, a natural problem is to characterize or classify biCayley graphs over a given group with certain valency and specific symmetric property. Some partial answers for this problem have been obtained. For example, in [16] Pisanski classified cubic bi-Cayley graphs over cyclic groups, and in [11], Kovács et al. gave a description of arc-transitive one-matching bi-Cayley graphs over abelian groups, from which one can obtain the classification of cubic arc-transitive one-matching bi-Cayley graphs over abelian groups. In [23], the automorphisms of the bi-Cayley graphs were investigated. In particular, some sufficient conditions for a bi-Cayley graph being vertextransitive or Cayley were given, and moreover, for a one-matching bi-Cayley graph $\Gamma$ over a group $H$, the normalizer of the group $H$ in $\operatorname{Aut}(\Gamma)$ was determined. By using this, a classification of cubic vertex-transitive bi-Cayley graphs over abelian groups was given. The facts listed above provide the motivation for us to consider the following problem.

Problem 1. Characterize cubic non-Cayley vertex-transitive bi-Cayley graphs over a $p$-group for an odd prime $p$.

Another motivation for us to consider this problem is: it is also related to the study of non-Cayley vertex-transitive graphs which is very active in 1980's. Let $p>3$ be a prime. It is easy to prove that every connected cubic non-Cayley vertex-transitive graph of order $2 p^{n}(n \geqslant 1)$ is a bi-Cayley graph over a $p$-group (see Lemma 9 ). So the above problem is equivalent to the problem of characterizing cubic non-Cayley vertex-transitive graphs of order $2 p^{n}$. By [5], every cubic symmetric graph of order $2 p^{n}$ ( $p>5$ is a prime) is a Cayley graph (see Proposition 4). Clearly, this is not true for the case when $p=5$ because the Petersen graph is non-Cayley. In fact, one may construct infinitely many cubic non-Cayley symmetric graphs of order $2 \cdot 5^{n}$ by considering the regular coverings of the Petersen graph.

It is known that for a prime $p$, every cubic non-Cayley vertex-transitive graph of order $2 p$ or $2 p^{2}$ is a generalized Petersen graph (see [14, 22]). In [12], the authors proved that every cubic non-Cayley vertex-transitive graph of order $2 p^{n}$, where $p>7$ is a prime and $n \leqslant p$, is a bi-Cayley graph over a $p$-group $P$ generated by two elements $a$ and $b$ of the same order and admitting an automorphism $\alpha \in \operatorname{Aut}(P)$ of order 4 such that $a^{\alpha}=b$ and $b^{\alpha}=a^{-1}$.

In this paper, we solve the above problem for the case when $P$ is a regular $p$-group where $p>5$ is a prime. It is proved that a connected cubic vertex-transitive bi-Cayley graph over a regular $p$-group $P$, where $p>5$ is a prime, is non-Cayley if and only if $\Gamma=\operatorname{BiCay}(P, R, L,\{1\})$ is 2-type, and $\operatorname{Cay}(P, R \cup L)$ is a tetravalent normal arctransitive Cayley graph with $\operatorname{Aut}(P, R \cup L) \cong \mathbb{Z}_{4}$. As an application, a classification of cubic non-Cayley vertex-transitive graphs of order $2 p^{3}$ is given for each prime $p$. 


\section{Preliminaries}

In this section, we shall introduce some notations, terminology and preliminary results. Let $n$ be a positive integer. Denote by $\mathbb{Z}_{n}$ the cyclic group of order $n$, by $\mathbb{Z}_{n}^{*}$ the multiplicative group of $\mathbb{Z}_{n}$ consisting of numbers coprime to $n$, and by $D_{2 n}$ the dihedral group of order $2 n$, respectively.

For a finite, simple and undirected graph $X$, we use $V(X), E(X), A(X)$ and $\operatorname{Aut}(X)$ to denote its vertex set, edge set, arc set and full automorphism group, respectively. For $u, v \in V(X), u \sim v$ means that $u$ is adjacent to $v$ and denote by $\{u, v\}$ the edge incident to $u$ and $v$ in $X$. For any subset $B$ of $V(X)$, the subgraph of $X$ induced by $B$ will be denoted by $X[B]$. A graph $X$ is said to be vertex-transitive, and arc-transitive (or symmetric) if $\operatorname{Aut}(X)$ acts transitively on $V(X)$ and $A(X)$, respectively.

Let $G$ be a permutation group on a set $\Omega$ and $\alpha \in \Omega$. Denote by $G_{\alpha}$ the stabilizer of $\alpha$ in $G$, that is, the subgroup of $G$ fixing the point $\alpha$. We say that $G$ is semiregular on $\Omega$ if $G_{\alpha}=1$ for every $\alpha \in \Omega$ and regular if $G$ is transitive and semiregular. Given a finite group $G$ and an inverse closed subset $S \subseteq G \backslash\{1\}$, the Cayley graph $\operatorname{Cay}(G, S)$ on $G$ with respect to $S$ is defined to have vertex set $G$ and edge set $\{\{g, s g\} \mid g \in G, s \in S\}$. A Cayley graph $\operatorname{Cay}(G, S)$ is connected if and only if $S$ generates $G$. Given a $g \in G$, define the permutation $R(g)$ on $G$ by $x \mapsto x g, x \in G$. Then $R(G)=\{R(g) \mid g \in G\}$, called the right regular representation of $G$, is a permutation group isomorphic to $G$. It is well-known that $R(G) \leqslant \operatorname{Aut}(\operatorname{Cay}(G, S))$. So, $\operatorname{Cay}(G, S)$ is vertex-transitive. In general, a vertex-transitive graph $X$ is isomorphic to a Cayley graph on a group $G$ if and only if its automorphism group has a subgroup isomorphic to $G$, acting regularly on the vertex set of $X$ (see [1, Lemma 16.3]).

A Cayley graph $\operatorname{Cay}(G, S)$ is said to be normal if $R(G)$ is normal in $\operatorname{Aut}(\operatorname{Cay}(G, S))$ (see [19]). Set $A=\operatorname{Aut}(\operatorname{Cay}(G, S))$ and $\operatorname{Aut}(G, S)=\left\{\alpha \in \operatorname{Aut}(G) \mid S^{\alpha}=S\right\}$.

Proposition 2. [19, Proposition 1.5] The Cayley graph $\operatorname{Cay}(G, S)$ is normal if and only if $A_{1}=\operatorname{Aut}(G, S)$, where $A_{1}$ is the stabilizer of the identity 1 of $G$ in $A$.

Let $p$ be a prime. A finite $p$-group $P$ is called a regular $p$-group if for any two elements $x$ and $y$ in $P$, there exist $c_{1}, c_{2}, \cdots, c_{r}$ in the derived group of $\langle x, y\rangle$ such that $(x y)^{p}=$ $x^{p} y^{p} c_{1}^{p} c_{2}^{p} \cdots c_{r}^{p}$.

Proposition 3. [6, Theorem 3.1] Let $p$ be a prime and $G$ a regular $p$-group with $p \neq$ 2,5. Let $X=\operatorname{Cay}(G, S)$ be a connected tetravalent Cayley graph on $G$. Then we have $\operatorname{Aut}(\operatorname{Cay}(G, S))=R(G) \rtimes \operatorname{Aut}(G, S)$.

Proposition 4. [5, Corollary 3.4] Let $p>5$ be a prime. Then every connected cubic symmetric graph of order $2 p^{n}$ is a Cayley graph.

The following proposition lists all of the tetravalent connected arc-transitive Cayley graphs of order $p^{3}$ for each prime $p$.

Proposition 5. [7, Theorem 4.1] Let $p$ be a prime and let $X=\operatorname{Cay}(G, S)$ be a tetravalent connected arc-transitive Cayley graph of order $p^{3}$. Then one of the following holds. 
(1) $G=\mathbb{Z}_{p^{3}}=\langle a\rangle, S=\left\{a, a^{-1}, a^{\lambda}, a^{-\lambda}\right\}\left(\lambda^{2} \equiv-1\left(\bmod p^{3}\right)\right)$.

(2) $G=\mathbb{Z}_{p^{2}} \times \mathbb{Z}_{p}=\langle a\rangle \times\langle b\rangle, S=\left\{a, a^{-1}, a^{\lambda} b,\left(a^{\lambda} b\right)^{-1}\right\}\left(\lambda^{2} \equiv-1(\bmod p)\right)$.

(3) $G=\mathbb{Z}_{p^{2}} \times \mathbb{Z}_{p}=\langle a\rangle \times\langle b\rangle, S=\left\{a, a^{-1}, a b,(a b)^{-1}\right\}$.

(4) $G=\left\langle a, b, c \mid a^{p}=b^{p}=c^{p}=1, c=[a, b],[a, c]=[b, c]=1\right\rangle, S=\left\{a, a^{-1}, b, b^{-1}\right\}$.

To end this section, we give some results regarding the bi-Cayley graphs. For the proof of these results, one may see [23]. Let $\Gamma=\operatorname{BiCay}(H, R, L, S)$ be a connected bi-Cayley graph over a group $H$. The following proposition gives some basic properties of $\Gamma$.

Proposition 6. The following hold.

(1) $H$ is generated by $R \cup L \cup S$.

(2) If $S \neq \emptyset$, then $S$ can be chosen to contain the identity element of $H$.

(3) For any automorphism $\alpha$ of $H, \operatorname{BiCay}(H, R, L, S) \cong \operatorname{BiCay}\left(H, R^{\alpha}, L^{\alpha}, S^{\alpha}\right)$.

Let $R(H)$ denote the right regular representation of $H$. Then $R(H)$ can be regarded as a group of automorphisms of $\operatorname{BiCay}(H, R, L, S)$ acting on its vertices by the rule

$$
h_{i}^{R(g)}=(h g)_{i}, \forall i \in \mathbb{Z}_{2}, h, g \in H .
$$

For an automorphism $\alpha$ of $H$, define two permutations on $V(\Gamma)=H_{0} \cup H_{1}$ as follows:

$$
\begin{array}{ll}
\delta_{\alpha}: & h_{0} \mapsto\left(h^{\alpha}\right)_{1}, h_{1} \mapsto\left(h^{\alpha}\right)_{0}, \forall h \in H, \\
\sigma_{\alpha}: & h_{0} \mapsto\left(h^{\alpha}\right)_{0}, h_{1} \mapsto\left(h^{\alpha}\right)_{1}, \forall h \in H .
\end{array}
$$

Set

$$
\begin{aligned}
& \mathrm{I}=\left\{\delta_{\alpha} \mid \alpha \in \operatorname{Aut}(H) \text { s.t. } R^{\alpha}=L, L^{\alpha}=R, S^{\alpha}=S^{-1}\right\}, \\
& \left.\mathrm{F}=\left\langle\sigma_{\alpha}\right| \alpha \in \operatorname{Aut}(H) \text { s.t. } R^{\alpha}=R, L^{\alpha}=L, S^{\alpha}=S\right\rangle .
\end{aligned}
$$

Proposition 7. Each element in $\mathrm{I} \cup \mathrm{F}$ is an automorphism of $\Gamma$. Furthermore, for any $\delta_{\alpha} \in \mathrm{I}$, if $\alpha$ has order 2 , then $\left\langle R(H), \delta_{\alpha}\right\rangle=R(H) \rtimes\left\langle\delta_{\alpha}\right\rangle$ acts regularly on $V(\Gamma)$.

Proposition 8. Let $\Gamma=\operatorname{BiCay}(H, R, L,\{1\})$ be a connected one-matching bi-Cayley graph over the group $H$. Then Aut $(\Gamma)$ contains a regular subgroup containing $R(H)$ if and only if there exists an automorphism $\alpha \in \operatorname{Aut}(H)$ of order at most 2 such that $R^{\alpha}=L$.

\section{Characterization}

Lemma 9. Let $p>3$ be a prime. Then every connected cubic non-Cayley vertex-transitive graph of order $2 p^{n}(n \geqslant 1)$ is a bi-Cayley graph over a p-group. 
Proof. Let $\Gamma$ be a cubic non-Cayley vertex-transitive graph of order $2 p^{n}$ with $p>3$ a prime. Set $A=\operatorname{Aut}(\Gamma)$. By Proposition 4 , either $\Gamma$ is non-symmetric or $\Gamma$ is symmetric and $p=5$. Let $P$ be a Sylow $p$-subgroup of $A$. If $\Gamma$ is non-symmetric, then the vertexstabilizer $A_{v}$ of $v \in V(\Gamma)$ is a 2-group, and so $P$ is semiregular on $V(\Gamma)$. If $\Gamma$ is symmetric and $p=5$, then $A_{v}$ is a $\{2,3\}$-group, and so $P$ is also semiregular on $V(\Gamma)$. Consequently, $P$ is always semiregular and has two orbits of equal size. This implies that $\Gamma$ must be a bi-Cayley graph over $P$.

Now we introduce the concept of quotient graph which will be used in the proof of the following lemma. Let $\Gamma$ be a connected vertex-transitive graph, and let $G \leqslant \operatorname{Aut}(\Gamma)$ be vertex-transitive on $\Gamma$. For a $G$-invariant partition $\mathcal{B}$ of $V(\Gamma)$, the quotient graph $\Gamma_{\mathcal{B}}$ is defined as the graph with vertex set $\mathcal{B}$ such that, for any two vertices $B, C \in \mathcal{B}, B$ is adjacent to $C$ if and only if there exist $u \in B$ and $v \in C$ which are adjacent in $\Gamma$. Let $N$ be a normal subgroup of $G$. Then the set $\mathcal{B}$ of orbits of $N$ in $V(\Gamma)$ is a $G$-invariant partition of $V(\Gamma)$. In this case, the symbol $\Gamma_{\mathcal{B}}$ will be replaced by $\Gamma_{N}$.

Lemma 10. Let $\Gamma=\operatorname{BiCay}(P, R, L, S)$ be a connected cubic non-Cayley vertex-transitive bi-Cayley graph over a regular $p$-group $P$, where $p>5$ is a prime. Let $A=\operatorname{Aut}(\Gamma)$. Then $R(P)$ is a normal Sylow p-subgroup of $A$.

Proof. By Proposition 4, $\Gamma$ must be non-symmetric. It follows that the vertex-stabilizer $A_{v}$ of any $v \in V(\Gamma)$ in $A$ is a 2-group. This implies that $A_{v} / A_{v}^{*} \leqslant \mathbb{Z}_{2}$, where $A_{v}^{*}$ is the kernel of $A_{v}$ acting on the neighborhood of $v$. Since $\Gamma$ is non-Cayley, one has $A_{v}>1$. If $A_{v} / A_{v}^{*}=1$, then $A_{v}\left(=A_{v}^{*}\right)$ fixes all neighbors of $v$, and by the vertex-transitivity and connectedness of $\Gamma$, we get that $A_{v}$ fixes all vertices of $\Gamma$, forcing that $A_{v}=1$, a contradiction. Thus, $A_{v} / A_{v}^{*} \cong \mathbb{Z}_{2}$, and so there is one and only one neighbor, say $u$, of $v$ such that $A_{u}=A_{v}$. By the arbitrariness of $v$, the following set

$$
\mathcal{B}=\left\{\{u, v\} \mid u, v \in V(\Gamma) \text { such that } A_{u}=A_{v}\right\} .
$$

is a 1 -factor of $\Gamma$. Clearly, for any $g \in A, A_{u^{g}}=A_{u}^{g}=A_{v}^{g}=A_{v^{g}}$. It follows that $\mathcal{B}$ is also an $A$-invariant partition of $V(\Gamma)$. Consider the quotient graph $\Gamma_{\mathcal{B}}$ of $\Gamma$ relative to $\mathcal{B}$, and let $K$ be the kernel of $A$ acting on $V\left(\Gamma_{\mathcal{B}}\right)$. Then $A / K$ is vertex-transitive on $\Gamma_{\mathcal{B}}$ and so $\Gamma_{\mathcal{B}}$ has regular valency. Since $\Gamma$ is cubic, $\Gamma_{\mathcal{B}}$ has valency at most 4 , and since $\left|\Gamma_{\mathcal{B}}\right|=|P|$ is odd, the valency of $\Gamma_{\mathcal{B}}$ is 2 or 4 . Since $p$ is odd and $K$ is a 2 -group, $R(P) \cong R(P) K / K$ must be regular on $V\left(\Gamma_{\mathcal{B}}\right)$.

Suppose $K \neq 1$. Then, $\mathcal{B}$ is the set of orbits of $K$. Since each orbit of $K$ is just an edge of $\Gamma$, the quotient graph $\Gamma_{\mathcal{B}}$ must be a cycle of length $p^{n}$, where $p^{n}=|P|$, and moreover, since $\Gamma$ has valency 3 , the edges between any two adjacent orbits of $K$ form a perfect matching. It follows that the neighbors of any $v \in V(\Gamma)$ are in three different orbits of $K$. Thus, $K_{v}$ fixes each neighbor of $v$. By the connectedness of $\Gamma$, we obtain that $K_{v}=1$ and hence $K \cong \mathbb{Z}_{2}$. As $R(P) K / K$ is regular on $V\left(\Gamma_{\mathcal{B}}\right), R(P) K=R(P) \times K$ is regular on $V(\Gamma)$, implying that $\Gamma$ is a Cayley graph, a contradiction.

Now we know that $K=1$. Then $A$ acts faithfully on $\mathcal{B}$, and so $A \leqslant \operatorname{Aut}\left(\Gamma_{\mathcal{B}}\right)$. Since $R(P)=R(P) K / K$ acts regularly on $V\left(\Gamma_{\mathcal{B}}\right), \Gamma_{\mathcal{B}}$ can be viewed as a Cayley graph on $P$. 
If $\Gamma_{\mathcal{B}}$ has valency 2 , then $\Gamma_{\mathcal{B}}$ is a cycle of length $p^{n}$ and $\operatorname{Aut}\left(\Gamma_{\mathcal{B}}\right) \cong D_{2 p^{n}}$. It follows that $|A|=2 p^{n}=2|P|$, contradicting that $A$ is not regular on $V(\Gamma)$. Thus, $\Gamma_{\mathcal{B}}$ has valency 4 . Since $P$ is a regular $p$-group with $p \neq 2,5$, by Proposition $3, \Gamma_{\mathcal{B}}$ is a normal Cayley graph on $P$. It follows that $R(P) \unlhd \operatorname{Aut}\left(\Gamma_{\mathcal{B}}\right)$, and since $A \leqslant \operatorname{Aut}\left(\Gamma_{\mathcal{B}}\right)$, one has $R(P) \unlhd A$, as required.

By Huppert [10, III, Theorem 10.2], a $p$-group of order $p^{n}$ with $n \leqslant p$ is regular. By Lemma 9 , the following corollary is straightforward.

Corollary 11. [12, Lemma 4.2] Let $\Gamma$ be a connected cubic vertex-transitive graph of order $2 p^{n}$, where $p>7$ is a prime and $n \leqslant p$. Then a Sylow $p$-subgroup of Aut $(\Gamma)$ is normal.

The following is the main result of this section.

Theorem 12. Let $\Gamma=\operatorname{BiCay}(P, R, L, S)$ be a connected cubic vertex-transitive bi-Cayley graph over a regular $p$-group $P$, where $p>5$ is a prime. Then $\Gamma$ is non-Cayley if and only if $\Gamma=\operatorname{BiCay}(P, R, L,\{1\})$ is 2-type, and $\operatorname{Cay}(P, R \cup L)$ is a tetravalent normal arc-transitive Cayley graph with $\operatorname{Aut}(P, R \cup L) \cong \mathbb{Z}_{4}$.

Proof. We first prove the sufficiency. Since $R=R^{-1}$ and $L=L^{-1}$, we may assume that $R=\left\{a, a^{-1}\right\}$ and $L=\left\{b, b^{-1}\right\}$. Since $\operatorname{Aut}(P, R \cup L) \cong \mathbb{Z}_{4}$, we may assume that $\operatorname{Aut}(P, R \cup L)=\langle\alpha\rangle$ such that $a^{\alpha}=b, b^{\alpha}=a^{-1}$. In particular, $\alpha$ interchanges $R$ and $L$. By the definition of $\delta_{\alpha}$ (see Eq 1)), $\delta_{\alpha}$ interchanges the two orbits of $R(P)$. It follows from Proposition 7 that $\Gamma$ is vertex-transitive. Suppose that $\Gamma$ is Cayley. Since $\Gamma=\operatorname{BiCay}(P, R, L,\{1\})$ is 2-type, by Proposition 8 there is an automorphism $\beta \in \operatorname{Aut}(P)$ of order at most 2 such that $R^{\beta}=L$. This implies that $\beta \in \operatorname{Aut}(P, R \cup L)=\langle\alpha\rangle$. Clearly, $R \neq L$, so $\beta$ has order 2 . It follows that $\beta=\alpha^{2}$. However, $R^{\alpha^{2}}=R$ and $L^{\alpha^{2}}=L$, a contradiction. Thus, $\Gamma$ is non-Cayley.

For the necessity, since $p$ is odd, the subgraph induced by each orbit of $R(P)$ must have even valency. It follows that $\Gamma$ is 0 - or 2 -type.

Case $1 \Gamma$ is 0-type.

By Lemma 10, $R(P) \unlhd \operatorname{Aut}(\Gamma)$. Since $R(P)$ has two orbits on $V(\Gamma)$, the quotient graph $\Gamma_{R(P)}$ of $\Gamma$ relative to $R(P)$ is the 3 -dipole Dip 3 (see Fig. (1)). This implies that

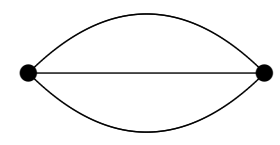

Figure 1: The 3-dipole Dip 3

$\Gamma$ is a regular cover of $\operatorname{Dip}_{3}$, and so $\operatorname{Aut}(\Gamma)$ can project to a subgroup of $\operatorname{Aut}\left(\Gamma_{R(P)}\right)$. Since $\operatorname{Aut}\left(\operatorname{Dip}_{3}\right) \cong S_{3} \times \mathbb{Z}_{2}$, Aut $(\Gamma) / R(P) \leqslant \mathbb{Z}_{2} \times \mathbb{Z}_{2}$ because $\Gamma$ is not symmetric by Proposition 4. Since $p>2$, $\operatorname{Aut}(\Gamma)=R(P) \rtimes Q$, where $1<Q \leqslant \mathbb{Z}_{2} \times \mathbb{Z}_{2}$ is a Sylow 
2-subgroup of $\operatorname{Aut}(\Gamma)$. As $\Gamma$ is vertex-transitive, there exists a 2-element, say $g$, such that $g$ interchanges the two orbits of $R(P)$. Since $Q \leqslant \mathbb{Z}_{2} \times \mathbb{Z}_{2}, g$ must be an involution. Therefore, $R(P) \rtimes\langle g\rangle$ acts regularly on $V(\Gamma)$, and so $\Gamma$ is Cayley. A contradiction occurs.

Case $2 \Gamma$ is 2-type.

Recall that $V(\Gamma)=P_{0} \cup P_{1}$ with $P_{0}=\left\{g_{0} \mid g \in P\right\}$ and $P_{1}=\left\{g_{1} \mid g \in P\right\}$. By Proposition 6 (2), we may assume that $S=\{1\}$. It follows that $\left\{g_{0}, g_{1}\right\} \in E(\Gamma)$ for each $g \in P$. Set $A=\operatorname{Aut}(\Gamma)$. Then $A$ is not regular on $V(\Gamma)$. So, for each $g \in P$, we have $A_{g_{0}} \neq 1$. Since $R(P) \unlhd A, A$ fixes the partition $V(\Gamma)=P_{0} \cup P_{1}$, and since $g_{1}$ is the unique neighbor of $g_{0}$ in $P_{1}$, it follows that $A_{g_{0}}$ fixes $g_{1}$. This implies that for any $\alpha \in A$, either $\left\{g_{0}, g_{1}\right\}^{\alpha}=\left\{g_{0}, g_{1}\right\}$ or $\left\{g_{0}, g_{1}\right\}^{\alpha} \cap\left\{g_{0}, g_{1}\right\}=\emptyset$. Set $\mathcal{B}=\left\{\left\{g_{0}, g_{1}\right\} \mid g \in P\right\}$. Then $A$ acts transitively on $\mathcal{B}$. Let $K$ be the kernel of $A$ acting on $\mathcal{B}$.

Suppose $K \neq 1$. Clearly, $\mathcal{B}$ is the set of orbits of $K$. Since each orbit of $K$ contains exactly one edge, the quotient graph $\Gamma_{\mathcal{B}}$ of $\Gamma$ relative to $\mathcal{B}$ must be a cycle of length $p^{n}$, where $p^{n}=|P|$. It follows that the neighbors of any $v \in V(\Gamma)$ are in three different orbits of $K$. Thus, $K_{v}$ fixes each neighbor of $v$. By the connectedness of $\Gamma$, we obtain that $K_{v}=1$ and hence $K \cong \mathbb{Z}_{2}$. Then $R(P) \times K$ is regular on $V(\Gamma)$, and so $\Gamma$ can be viewed as a Cayley graph on $R(P) \times K$. This is impossible.

Now assume that $K=1$. Then $A$ acts faithfully on $\mathcal{B}$. It follows that $A \leqslant \operatorname{Aut}\left(\Gamma_{\mathcal{B}}\right)$. It is easy to see that $R(P)$ is regular on $\mathcal{B}$, and so $\Gamma_{\mathcal{B}}$ can be viewed as a Cayley graph on $P$. Recall that $\Gamma=\operatorname{BiCay}(P, R, L,\{1\})$. Set $R=\{a, b\}$ and $L=\{x, y\}$.

Suppose $|R \cap L|=2$. Then $R=L$. In this case, it is easy to see that the permutation $\alpha=\prod_{g \in P}\left(g_{0} g_{1}\right)$ is an automorphism of $\Gamma$. Furthermore, $\alpha$ commutes with $R(P)$. So, $R(P) \times\langle\alpha\rangle$ acts regularly on $V(\Gamma)$, a contradiction.

Suppose $|R \cap L|=1$. Without loss of generality, assume that $a=x$. Then $P=\langle a, b, y\rangle$. Since $R^{-1}=R$ and $L^{-1}=L$, all $a, b, x, y$ are involutions. This is clearly impossible because $P$ is a $p$-group with $p>2$.

Suppose $|R \cap L|=0$. In this case, the neighbors of $\left\{1_{0}, 1_{1}\right\}$ in $\Gamma_{\mathcal{B}}$ are $\left\{a_{0}, a_{1}\right\},\left\{b_{0}, b_{1}\right\}$, $\left\{x_{0}, x_{1}\right\}$ and $\left\{y_{0}, y_{1}\right\}$. So, $\Gamma_{\mathcal{B}}$ is a tetravalent Cayley graph on $P$. Note that $A_{1_{0}}=A_{1_{1}}$. Since $A$ is not regular on $V(\Gamma), A_{1_{0}}$ interchanges $a_{0}$ and $b_{0}$, and also interchanges $x_{1}$ and $y_{1}$. Since $\left\{1_{0}, 1_{1}\right\}$ is a block of $A, A$ contains an element interchanging $1_{0}$ and $1_{1}$. This implies that $A_{\left\{1_{0}, 1_{1}\right\}}$ is transitive on the neighborhood of $\left\{1_{0}, 1_{1}\right\}$ in $\Gamma_{\mathcal{B}}$. So, $A$ is an arc-transitive automorphism group of $\Gamma_{\mathcal{B}}$. Let $X=\operatorname{Cay}(P, R \cup L)$. It is easy to verify that the following map

$$
f: g \mapsto\left\{g_{0}, g_{1}\right\}, \forall g \in P
$$

is an isomorphism from $X$ to $\Gamma_{\mathcal{B}}$. So, $X \cong \Gamma_{\mathcal{B}}$. Since $\Gamma_{\mathcal{B}}$ is arc-transitive, $X$ is also arc-transitive. Since $P$ is a $p$-group with $p>2$, we may assume that $R=\left\{a, a^{-1}\right\}$ and $L=\left\{x, x^{-1}\right\}$. By Proposition $3, X$ is a normal Cayley graph, and so $\operatorname{Aut}\left(\Gamma_{\mathcal{B}}\right)=$ $R(P) \rtimes \operatorname{Aut}(P, R \cup L)$. Clearly, $\operatorname{Aut}(P, R \cup L)$ acts faithfully on $R \cup L$. Since $p>2$, it is easy to see that $R, L$ are blocks of $\operatorname{Aut}(P, R \cup L)$ on $R \cup L$. It follows that $\operatorname{Aut}(P, R \cup L) \leqslant D_{8}$, and so $A \leqslant \operatorname{Aut}\left(\Gamma_{\mathcal{B}}\right) \leqslant R(P) \rtimes D_{8}$. Also, as $R, L$ are blocks of $\operatorname{Aut}(P, R \cup L)$ on $R \cup L$, by Proposition 7, each element in $\operatorname{Aut}(P, R \cup L)$ can also induce an automorphism of $\Gamma$. This implies that $A=\operatorname{Aut}\left(\Gamma_{\mathcal{B}}\right)$. Since $\Gamma$ is arc-transitive, $\operatorname{Aut}(P, R \cup L)$ is isomorphic to $\mathbb{Z}_{2} \times \mathbb{Z}_{2}, \mathbb{Z}_{4}$, or $D_{8}$. 
If $\operatorname{Aut}(P, R \cup L) \cong \mathbb{Z}_{2} \times \mathbb{Z}_{2}$ or $D_{8}$, then there exists an involution, say $\alpha$, in $\operatorname{Aut}(P, R \cup L)$ which interchanges $R$ and $L$. By Proposition 7, $\alpha$ can induce an automorphism, say $\delta_{\alpha}$, of $\Gamma$ of order 2 such that $R(P) \rtimes\left\langle\delta_{\alpha}\right\rangle$ is regular on $V(\Gamma)$, and so $\Gamma$ is Cayley, a contradiction. Thus, $\operatorname{Aut}(P, R \cup L) \cong \mathbb{Z}_{4}$.

\section{Cubic non-Cayley vertex-transitive graphs of order $2 p^{3}$}

Let $p$ be an odd prime. We first introduce some cubic connected non-Cayley vertextransitive graphs of order $2 p^{3}$. It is well known that $\mathbb{Z}_{p^{n}}^{*}$ is cyclic and has order $p^{n-1}(p-1)$. So, if $4 \mid(p-1)$ then $\mathbb{Z}_{p^{n}}^{*}$ has a unique subgroup of order 4 . Clearly, if $\lambda$ is an element of order 4 in $\mathbb{Z}_{p^{n}}^{*}$, then $\{1,-1, \lambda,-\lambda\}$ is the unique subgroup of order 4 in the cyclic group $\mathbb{Z}_{p^{n}}^{*}$

Example 13. Let $p$ be a prime such that $p-1$ is divisible by 4 and let $\lambda$ be an element of order 4 in $\mathbb{Z}_{p^{3}}^{*}$. The graph $\mathcal{N C}_{2 p^{3}}^{0}$ is defined to be the bi-Cayley graph $\operatorname{BiCay}\left(\mathbb{Z}_{p^{3}}, R, L,\{1\}\right)$, where $\mathbb{Z}_{p^{3}}=\langle a\rangle, R=\left\{a, a^{-1}\right\}$ and $L=\left\{a^{\lambda}, a^{-\lambda}\right\}$.

By the uniqueness of the subgroup of order 4 in $\mathbb{Z}_{p^{3}}$, the graph $\mathcal{N C}_{2 p^{3}}^{0}$ is independent of the choice of $\lambda$. Let $\alpha$ be the automorphism of $\mathbb{Z}_{p^{3}}$ induced by the map $a \mapsto a^{\lambda}$. Then $\alpha$ swaps $R$ and $L$, and by Proposition $7, \delta_{\alpha} \in \operatorname{Aut}\left(\mathcal{N C}_{2 p^{3}}^{0}\right)$ (see Equations (1)-(2) for the definition of $\left.\delta_{\alpha}\right)$ and so $\mathcal{N} \mathcal{C}_{2 p^{3}}^{0}$ is vertex-transitive because $\delta_{\alpha}$ swaps the two orbits of $\mathbb{Z}_{p^{3}}$ on $V\left(\mathcal{N C}_{2 p^{3}}^{0}\right)$.

In view of $[20$, Theorem 1$]$, we have $\operatorname{Cay}\left(\mathbb{Z}_{p^{3}}, R \cup L\right)$ is a tetravalent normal arctransitive Cayley graph and $\operatorname{Aut}\left(\mathbb{Z}_{p^{3}}, R \cup L\right) \cong \mathbb{Z}_{4}$. If $p=5$, then by Magma [3], $\mathcal{N C}_{2 p^{3}}^{0}$ is non-Cayley vertex-transitive and $\left|\operatorname{Aut}\left(\mathcal{N C}_{2 p^{3}}^{0}\right)\right|=4 p^{3}$, and if $p>5$, then by Theorem 12, again we have $\mathcal{N C}_{2 p^{3}}^{0}$ is non-Cayley vertex-transitive.

Example 14. Let $p$ be a prime such that $p-1$ is divisible by 4 and let $\lambda$ be an element of order 4 in $\mathbb{Z}_{p}^{*}$. The graph $\mathcal{N C}_{2 p^{3}}^{1}$ is defined to be the bi-Cayley graph $\operatorname{BiCay}\left(\mathbb{Z}_{p^{2}} \times\right.$ $\left.\mathbb{Z}_{p}, R, L,\{1\}\right)$, where $\mathbb{Z}_{p^{2}} \times \mathbb{Z}_{p}=\langle a\rangle \times\langle b\rangle, R=\left\{a, a^{-1}\right\}$ and $L=\left\{(a b)^{\lambda},(a b)^{-\lambda}\right\}$.

By the uniqueness of the subgroup of order 4 in $\mathbb{Z}_{p}$, the graph $\mathcal{N C}_{2 p^{3}}^{1}$ is independent of the choice of $\lambda$. Let $\beta$ be the automorphism of $\mathbb{Z}_{p^{2}} \times \mathbb{Z}_{p}$ induced by the map $a \mapsto$ $(a b)^{\lambda}, b \mapsto a^{\lambda^{3}+\lambda} b^{-\lambda}$. Then $\beta$ swaps $R$ and $L$, and by Proposition $7, \delta_{\beta} \in \operatorname{Aut}\left(\mathcal{N C}_{2 p^{3}}^{1}\right)$ and so $\mathcal{N C}_{2 p^{3}}^{1}$ is vertex-transitive because $\delta_{\beta}$ swaps the two orbits of $\mathbb{Z}_{p^{2}} \rtimes \mathbb{Z}_{p}$ on $V\left(\mathcal{N} \mathcal{C}_{2 p^{3}}^{1}\right)$.

In view of $\left[21\right.$, Proposition 3.3], we have Cay $\left(\mathbb{Z}_{p^{2}} \times \mathbb{Z}_{p}, R \cup L\right)$ is a tetravalent normal arc-transitive Cayley graph and $\operatorname{Aut}\left(\mathbb{Z}_{p^{2}} \times \mathbb{Z}_{p}, R \cup L\right) \cong \mathbb{Z}_{4}$. If $p=5$, then by Magma [3], $\mathcal{N C}_{2 p^{3}}^{1}$ is non-Cayley vertex-transitive and $\left|\operatorname{Aut}\left(\mathcal{N C}_{2 p^{3}}^{1}\right)\right|=4 p^{3}$, and if $p>5$, then by Theorem 12, again we have $\mathcal{N C}_{2 p^{3}}^{1}$ is non-Cayley vertex-transitive.

Also, note that $\operatorname{Aut}\left(\mathcal{N C}_{2 p^{3}}^{0}\right) \cong \mathbb{Z}_{p^{3}} \rtimes \mathbb{Z}_{4}$ and $\operatorname{Aut}\left(\mathcal{N C}_{2 p^{3}}^{1}\right) \cong\left(\mathbb{Z}_{p^{2}} \rtimes \mathbb{Z}_{p}\right) \rtimes \mathbb{Z}_{4}$. It follows that $\mathcal{N C}_{2 p^{3}}^{0}$ and $\mathcal{N} \mathcal{C}_{2 p^{3}}^{1}$ are not isomorphic to each other.

Theorem 15. Let $p$ be a prime. Then a cubic vertex-transitive graph of order $2 p^{3}$ is non-Cayley if and only if it is isomorphic to $\mathcal{N C}_{2 p^{3}}^{0}$ or $\mathcal{N C}_{2 p^{3}}^{1}$. 
Proof. By [15], all connected cubic vertex-transitive graphs of order 16 are Cayley. By [17], if $p=3$, then all connected cubic vertex-transitive graphs of order 54 are Cayley, and if $p=5$, then up to isomorphism, there are exactly two non-Cayley vertex-transitive graphs of order $2 \cdot 5^{3}$, and so $\Gamma \cong \mathcal{N C}_{2 \cdot 5^{3}}^{0}$ or $\mathcal{N C}_{2 \cdot 5^{3}}^{1}$.

In what follows, assume that $p>5$. By Lemma $9, \Gamma$ is a bi-Cayley graph over a group $P$, where $P$ is a Sylow $p$-subgroup of $\operatorname{Aut}(\Gamma)$. By Theorem $12, \Gamma=\operatorname{BiCay}(P, R$, $L,\{1\})$ and $\operatorname{Cay}(P, R \cup L)$ is a tetravalent normal arc-transitive Cayley graph such that $\operatorname{Aut}(P, R \cup L) \cong \mathbb{Z}_{4}$. Since $|\Gamma|=2 p^{3}$, one has $|P|=p^{3}$. Noting that $R=R^{-1}$ and $L=L^{-1}$, by Proposition 5 , one of the following happens:

(1) $P=\mathbb{Z}_{p^{3}}=\langle a\rangle, R=\left\{a, a^{-1}\right\}, L=\left\{a^{\lambda}, a^{-\lambda}\right\}\left(\lambda^{2} \equiv-1\left(\bmod p^{3}\right)\right) ;$

(2) $P=\mathbb{Z}_{p^{2}} \times \mathbb{Z}_{p}=\langle a\rangle \times\langle b\rangle, R=\left\{a, a^{-1}\right\}, L=\left\{(a b)^{\lambda},(a b)^{-\lambda}\right\}\left(\lambda^{2} \equiv-1(\bmod p)\right)$;

(3) $P=\mathbb{Z}_{p^{2}} \times \mathbb{Z}_{p}=\langle a\rangle \times\langle b\rangle, R=\left\{a, a^{-1}\right\}, L=\left\{a b,(a b)^{-1}\right\}$;

(4) $P=\left\langle a, b, c \mid a^{p}=b^{p}=c^{p}=1, c=[a, b],[a, c]=[b, c]=1\right\rangle$, $R=\left\{a, a^{-1}\right\}, L=\left\{b, b^{-1}\right\}$.

If (1) happens, then $\Gamma \cong \mathcal{N C}_{2 p^{3}}^{0}$, and if (2) happens, then $\Gamma \cong \mathcal{N C}_{2 p^{3}}^{1}$. If (3) happens, then in view of $\left[21\right.$, Proposition 3.3], we have $\operatorname{Aut}(P, R \cup L) \cong \mathbb{Z}_{2} \times \mathbb{Z}_{2}$. This is impossible by Theorem 12. If (3) happens, then $P=\langle a, b, c| a^{p}=b^{p}=c^{p}=1, c=[a, b],[a, c]=[b, c]=$ $1\rangle$, and any two elements generating $P$ have the same relation as $a$ and $b$. It follows that $\operatorname{Aut}(P, R \cup L) \cong D_{8}$, and by Theorem $12, \Gamma$ is not non-Cayley, a contradiction.

\section{Acknowledgements}

This work was supported by the National Natural Science Foundation of China (11271012, 11571035, 11231008), the Fundamental Research Funds for the Central Universities (2015JBM110) and the 111 Project of China (B16002). The author also would like to thank the anonymous referee for the valuable comments and suggestions.

\section{References}

[1] N. Biggs, Algebraic Graph Theory, Second ed, Cambridge University Press, Cambridge, 1993.

[2] J.A. Bondy, U.S.R. Murty, Graph Theory with Applications, New York: Elsevier North Holland, 1976.

[3] W. Bosma, C. Cannon, C. Playoust, The MAGMA algebra system I: The user language, J. Symbolic Comput. 24 (1997) 235-265.

[4] M.J. de Resmini, D. Jungnickel, Strongly regular semi-Cayley graphs, J. Algebraic Combin. 1 (1992) 171-195.

[5] Y.-Q. Feng, J.H. Kwak, Cubic symmetric graphs of order twice an odd prime power, J. Aust. Math. Soc. 81 (2006), 153-164. 
[6] Y.-Q. Feng, M.Y. Xu, Automorphism groups of tetravalent Cayley graphs on regular p-groups, Discrete Math. 305 (2005) 354-360.

[7] Y.-Q. Feng, M.Y. Xu, Normality of tetravalent Cayley graphs of odd prime-cube order and its application, Acta Math. Sin., English Ser. 21 (2005) 903-912.

[8] X. Gao, W. Liu, Y. Luo, On the extendability of certain semi-Cayley graphs of finite abelian groups, Discrete Math. 311 (2011) 1978-1987.

[9] X. Gao, Y. Luo, The spectrum of semi-Cayley graphs over abelian groups, Linear Algebra Appl. 432 (2010) 2974-2983.

[10] B. Huppert, Eudliche Gruppen I, Springer-Verlag, Berlin, 1967.

[11] I. Kovács, A. Malnič, D. Marušič, Š. Miklavič, One-matching bi-Cayley graphs over abelian groups, European J. Combin. 30 (2009) 602-616.

[12] K. Kutnar, D. Marušič, C. Zhang, On cubic non-Cayley vertex-transitive graphs, J. Graph Theory 69 (2012) 77-95.

[13] K.H. Leung, S.L. Ma, Partial difference triples, J. Algebraic Combin. 2 (1993) 397409.

[14] D. Marušič, On vertex symmetric digraphs, Discrete Math. 36 (1981) 69-81.

[15] B.D. McKay, Transitive graphs with fewer than 20 vertices, Math. Comp. 33 (1979) 1101-1121.

[16] T. Pisanski, A classication of cubic bicirculants, Discrete Math. 307 (2007) 567-578.

[17] P. Potočnik, P. Spiga, G. Verret, A census of small connected cubic vertex-transitive graphs, http://www.matapp.unimib.it/ spiga/.

[18] H. Wielandt, Finite Permutation Groups, Academic Press, New York, 1964.

[19] M.Y. Xu, Automorphism groups and isomorphisms of Cayley digraphs, Discrete Math. 182 (1998) 309-319.

[20] M.Y. Xu, A note on one-regular graphs, Chinese Sci. Bull. 45 (2000) 2160-2162.

[21] J. Xu, M.Y. Xu, Arc-transitive Cayley graphs of valency at most four on abelian groups, Southeast Asian Bull. Math. 25 (2001) 355-363.

[22] J.-X. Zhou, Cubic vertex-transitive graphs of order $2 p^{2}$, Advances in Math. (China) 37 (2008) 605-609.

[23] J.-X. Zhou, Yan-Quan Feng, Cubic bi-Cayley graphs over abelian groups, European J. Combin. 36 (2014) 679-693. 